\title{
Effects of the electron-phonon coupling near and within the insulating Mott phase
}

\author{
C.A. Perroni, V. Cataudella, G. De Filippis, and V. Marigliano Ramaglia \\ Coherentia-INFM and Dipartimento di Scienze Fisiche, \\ Università degli Studi di Napoli "Federico II", \\ Complesso Universitario Monte Sant'Angelo, \\ Via Cintia, I-80126 Napoli, Italy
}

(Dated: March 22, 2021)

\begin{abstract}
The role of the electron-phonon interaction in the Holstein-Hubbard model is investigated in the metallic phase close to the Mott transition and in the insulating Mott phase. The model is studied by means of a variational slave boson technique. At half-filling, mean-field static quantities are in good agreement with the results obtained by numerical techniques. By taking into account gaussian fluctuations, an analytic expression of the spectral density is derived in the Mott insulating phase showing that an increase of the electron-phonon coupling leads to a sensitive reduction of the Mott gap through a reduced effective repulsion. The relation of the results with recent experimental observations in strongly correlated systems is discussed.
\end{abstract}

\section{INTRODUCTION}

The understanding of the properties of Mott insulators such as $\mathrm{CaTiO}_{3}$ and $\mathrm{V}_{2} \mathrm{O}_{3}$ represents a long-standing

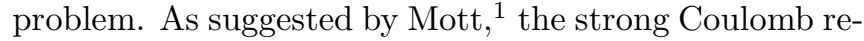
pulsion among the electrons can cause a metal-insulator transition opening a gap in the density of states that is usually known as Mott gap. The correlation-driven metal-insulator transition is often investigated within the framework of the Hubbard lattice Hamiltonian which takes into account the electron interaction through the on-site repulsion term $U$. As developed by Hubbard, ${ }^{2}$ two subbands generically called lower and upper Hubbard bands separated by the Mott gap of the order of the energy $U$ arise in the excitation spectrum for large enough repulsion.

The correlation effects are important not only on the insulating side but also on the metallic phase where a great deal of insight has been obtained by using the Gutzwiller wave function ${ }^{3}$ A progress toward the understanding of the metal-insulator transition has been made by the reformulation of the problem in terms of slave bosons,$\frac{4}{\underline{y}}$ which reproduces the Gutzwiller approximation at the saddle-point level and allows to study the effects of gaussian fluctuations in the metallic ${ }^{\frac{5}{2}}$ and in the insulating phase $\underline{\underline{6}}$ In this state the resulting single-particle spectral function consists of two broad incoherent contributions reminiscent of the lower and upper Hubbard bands. An overall description of the Mott transition in the Hubbard model can be obtained by means of the dynamical mean field theory $(D M F T)$ that is exact in the limit of infinite dimension $\underline{\underline{z}}$ The study of the infinite dimensional model has essentially confirmed the validity of the description obtained when slave boson fluctuations about the mean-field $(M F)$ are considered.

Although the Hubbard Hamiltonian captures the fundamental properties of systems near the Mott transition, it does not take into account the role of the lattice degrees of freedom. Actually, the presence of strong electronphonon $(e l-p h)$ interactions has been pointed out in several systems, such as cuprates, $\stackrel{8.9}{,}$ manganites,$\frac{10}{,}$ and
$V_{2} \mathrm{O}_{3} \stackrel{11}{11}$. In addition to the on-site repulsion, the most natural model incorporates a short-range interaction of the electrons with local phonon modes of constant frequency $\omega_{0} \frac{12}{2}$ This model is described by the HolsteinHubbard Hamiltonian $H$

$$
\begin{aligned}
H= & -t \sum_{<i, j>, \sigma} c_{i \sigma}^{\dagger} c_{j \sigma}+U \sum_{i} n_{i \uparrow} n_{i \downarrow}+ \\
& \omega_{0} \sum_{i} a_{i}^{\dagger} a_{i}+g \omega_{0} \sum_{i} n_{i}\left(a_{i}+a_{i}^{\dagger}\right) .
\end{aligned}
$$

Here $t$ is the electron transfer integral between nearest neighbor sites $\langle i, j\rangle$ of a $d$-dimensional simple cubic lattice, $c_{i \sigma}^{\dagger}\left(c_{i \sigma}\right)$ creates (destroys) an electron with spin $\sigma$ at the site $i$ and $n_{i}=\sum_{\sigma} n_{i \sigma}=\sum_{\sigma} c_{i \sigma}^{\dagger} c_{i \sigma}$ is the local density operator. In Eq.(1) $a_{i}^{\dagger}\left(a_{i}\right)$ is the creation (annihilation) phonon operator at the site $\mathrm{i}$, and the parameter $g$ represents the coupling constant between electrons and local displacements. The dimensionless parameter $\lambda=g^{2} \omega_{0} / W$, with $W=2 d t$ bare electron half bandwidth, is typically used to measure the strength of the $e l-p h$ interaction in the adiabatic regime characterized by small values of the ratio $\gamma=\omega_{0} / t$. Within this regime the Coulomb repulsion is found to dominate the properties of the metallic phase also with a sizable $e l-p h$ coupling $\frac{13}{13}$ Furthermore, there is a very little softening of the phonon frequency on the approach to the Mott transition since the Hubbard term $U$ suppresses charge

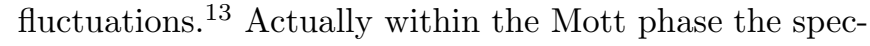
tral density shows phonon side bands whose peaks are separated by multiples of the bare frequency $\omega_{0} \underline{14} \mathrm{Fi}$ nally, close to the Mott transition at finite density, an intermediate $e l-p h$ coupling leads to the phase separation between a metal and an insulator 15

In this paper the study of the Holstein-Hubbard model focuses on the role of the $e l-p h$ interaction in modifying the physical properties of the electrons close to the metal-insulator transition and in the insulating Mott phase. The starting point of the approach is the gener- 
alized Lang-Firsov transformation ${ }^{16} U=e^{V}$, with

$$
V=g \sum_{i}\left[<n_{i}>+f_{i}\left(n_{i}-<n_{i}>\right)\right]\left(a_{i}-a_{i}^{\dagger}\right),
$$

where the parameters $f_{i}$ take into account the polaronic local density fluctuations which couple to the lattice distortions. The next step is the functional-integral representation in terms of the four-slave bosons $e_{i}, p_{i \sigma}$, and $d_{i}$ obtained by imposing the equivalence with the original model through the Lagrange multipliers $\lambda_{i}^{(1)}$ and $\lambda_{i \sigma}^{(2)} \underline{\underline{4}}$ First we consider the $M F$ solution at half-filling, then slave boson gaussian fluctuations on the top of the $M F$ finding that the resulting Mott gap is sensitively reduced with increasing $\lambda$ since it is determined by the $e l-e l$ repulsion renormalized by the effects of the $e l-p h$ coupling. Finally the relation of the results with recent experimental observations in strongly correlated systems is discussed.

The $M F$ solution in the paramagnetic homogeneous phase is obtained by replacing the Bose fields with their mean values $\left(\left\langle e_{i}\right\rangle=e_{0},\left\langle d_{i}\right\rangle=d_{0},\left\langle p_{i \sigma}\right\rangle=p_{0}\right)$ and by assuming $f_{i}=f, \lambda_{i}^{(1)}=\lambda_{0}^{(1)}$, and $\lambda_{i \sigma}^{(2)}=\lambda_{0}^{(2)} 17$ The resulting mean-field Hamiltonian is characteristic of free fermion quasi-particles and phonons and the mean double occupation is controlled by $U_{\text {eff }}=U+2 g^{2} \omega_{0}\left(f^{2}-2 f\right)$.

At half-filling the $M F$ energy per site $u$ is given by a simple functional depending on $f$ and $d_{0}$

$$
u=q e^{-f^{2} g^{2}} \bar{\varepsilon}+U_{e f f} d_{0}^{2}-g^{2} \omega_{0},
$$

with $q=8 d_{0}^{2}-16 d_{0}^{4}$ and $\bar{\varepsilon}$ mean bare kinetic energy. The Mott phase is the insulating state for large $U_{\text {eff }}>0$ characterized by $q=0, d_{0}=0$, and the energy per site equal to $-g^{2} \omega_{0}$ (characteristic energy of the limit $U / t=$ $\infty)$. When $U_{\text {eff }}$ becomes negative, a on-site bipolaron solution sets in corresponding to $d_{0}^{2}=0.5, f=1$, and energy $u=U / 2-2 g^{2} \omega_{0}$.

In Fig. 1 the phase diagram at half-filling is reported for $\gamma=0.2$ in the three-dimensional case. By analyzing the behavior of $d_{0}$, it is found that the transition to $M I$ is always found to be second order, that to the bipolaronic insulator $(B I)$ is first order, finally that between $M I$ and $B I$ is again discontinuous in excellent agreement with the results derived by the $D M F T$ solution ${ }^{13}$ In particular we notice that, with increasing $\lambda$, the line separating the $M$ and $M I$ phases shifts to values of $U$ larger than $U_{c}$, the critical value in absence of the $e l-p h$ coupling. Actually the Mott transition is influenced by the $e l-p h$ interaction since it is $U_{\text {eff }}$ that governs the transition and it becomes smaller with increasing $\lambda$. Therefore, the condition $U_{\text {eff }} \simeq U_{c}$, characteristic of a transition driven by the $e l-e l$ interaction, implies that the transition occurs for larger values of the bare $U$. Within the $M F$ approach the interplay between $e l-e l$ and $e l-p h$ interactions in affecting the Mott phase is essentially linked to the value of the parameter $f$ that, at fixed adiabaticity ratio and $\lambda$, is weakly decreasing with increasing $U$ and it is of the order of $\gamma / 4 d$ in the adiabatic regime near the Mott transition. The transition line between $M$ and $M I$ phase is given by $\lambda \simeq\left(U-U_{c}\right) /(2 f W) \simeq\left(U-U_{c}\right) / \gamma t$. Therefore, as shown in the inset of Fig. 1, the dependence of the Mott transition line on $\lambda$ becomes also more pronounced with increasing the adiabaticity ratio $\gamma$. In the atomic limit $(\gamma=\infty)$ we recovers the exact solution without metallic phase with $f=1$ and $U_{c}=0$ (dotted line in Fig. 1 corresponding to $\lambda=U / 2 W)$.

While the Mott transition is driven by the growth of the spin susceptibility, the transition from $M$ to $B I$ is characterized by the enhancement of the charge fluctuations inducing a decrease of the effective repulsion. In Fig. 2 we report the $M F$ results for the spectral weight $Z$ at the Fermi energy (equal to $\mathrm{m} / \mathrm{m}^{*}$ ) and the local magnetic moment $M=<\left(M_{i}^{z}\right)^{2}>$ stressing the effects of the $e l-p h$ interaction. Within the $M F$ approach we simply have $Z=q e^{-f^{2} g^{2}}$ and $M=1-2 d_{0}$. Far from the Mott transition $\left(U / U_{c}\right.$ small $) Z$ decreases with increasing the $e l-p h$ coupling as expected for any localizing interaction. However, near the Mott phase, the effects due to the reduction of $U_{\text {eff }}$ become more marked and are able to induce the enhancement of $Z$ with increasing $\lambda$ that, again, is in good agreement with DMFT results 13 In Fig. 2 we also show that the $M$ phase is reduced in comparison with its value at $\lambda=0$ for any ratio $U / U_{c}$ implying that the double occupation $d_{0}$ increases by approaching the $B I$ phase. Therefore, while $M$ increases as function of $U$, it decreases as function of $\lambda$.

The $M F$ solution can be readily generalized at densities different from half-filling. Within the $M F$ approach some of us have shown that the interplay of strong local $e l-e l$ and $e l-p h$ interactions can push the system toward a phase separation between states characterized by different lattice distortions $\stackrel{17}{ }$ The phase coexistence occurs for intermediate values of the $e l-p h$ coupling and its relevance within the Hubbard-Holstein model has been confirmed also by DMFT works $\underline{15}$

The task of including charge fluctuations described by the $e$ and $d$ fields is simplified in the Mott phase by the fact that at $M F$ level the Bose fields $e$ and $d$ are not condensed $\left(e_{0}=d_{0}=0\right)$, while $p_{0}=1 / \sqrt{2} \underline{\underline{6}}$ At gaussian level the fluctuations of the $e$ and $d$ fields are actually decoupled not only from those of the $p$ fields but also from the phononic $a$ fields. Clearly the matrix elements of the fluctuating fields $e$ and $d$ are renormalized by $e l-p h$ contributions because of $U_{\text {eff }}$. The procedure of calculation is the following: we start at densities different from half-filling $(n<1)$, then we calculate the quantities in the limit $n \rightarrow 1^{-}$for values of the parameters where the system is in the Mott phase at half-filling $\underline{\underline{6}}$

The inclusion of gaussian fluctuations allows to calculate the one-particle spectral function $A(\omega)$ of the insulating Mott phase in the limit $n \rightarrow 1^{-}$(at $M F$ it is identically zero). The incoherent contribution arises from the complicated motion of a quasi-particle surrounded by the cloud of charge and lattice excitations that it leaves behind. In the limit $n \rightarrow 1^{-}$it is possible to derive an 
analytic expression of the spectral function $A(\omega)$ yielding at zero temperature

$$
\begin{aligned}
A(\omega)= & e^{-g^{2} f^{2}} \tilde{A}(\omega)+ \\
& e^{-g^{2} f^{2}} \sum_{n=1}^{\infty} \frac{\left(g^{2} f^{2}\right)^{n}}{n !} \theta\left(-\omega-n \omega_{0}\right) \tilde{A}\left(\omega+n \omega_{0}\right)+ \\
& e^{-g^{2} f^{2}} \sum_{n=1}^{\infty} \frac{\left(g^{2} f^{2}\right)^{n}}{n !} \theta\left(\omega-n \omega_{0}\right) \tilde{A}\left(\omega-n \omega_{0}\right),
\end{aligned}
$$

with $\theta(x)$ Heaviside function and the function $\tilde{A}(\omega)$ akin to that obtained for the Hubbard model with $U=U_{\text {eff }}$,

The first term of Eq. (5) is the product of two quantities, with $e^{-f^{2} g^{2}}$ renormalization factor due to $e l-p h$ coupling. Through $\tilde{A}(\omega)$ this term is able to reconstruct lower and upper Hubbard bands. The second and the third term in Eq. (5) represent the contribution due to the phonon replicas of hole- and particle-type, respectively 18 In Fig. 3(a) we report the spectral density $N(\omega)=A(\omega) / 2 \pi$ (solid line) together with the resulting first term of Eq. (5) (dotted line) and the contribution from the phonon replicas (dashed line). We notice that this last term provides a non negligible spectral weight to the total spectral density at energies out of the gap of $\tilde{A}(\omega)$. Therefore, the Mott gap of the system is determined by the gap of the function $\tilde{A}(\omega)$ and it is simply given by $\Delta=U_{\text {eff }} \xi$. As seen in Fig. 3(b), the reduction of the gap with increasing $\lambda$ can be also of the order of bare half bandwidth $W \stackrel{19}{\underline{19}}$ Finally we stress that the calculated gap is traceable to the difference of the chemical potentials at $n=1$ and $n=1^{-}$, in analogy with the results of the Hubbard model $\underline{6}$ For $n=1$ we have $\mu(1)=U / 2-2 g^{2} \omega_{0}$, while for $n=1^{-}$the chemical potential is $\mu\left(1^{-}\right)=\mu(1)-U_{\text {eff }} \xi / 2$. Since the system has particle-hole symmetry, the gap $\Delta$ can be obtained as $\Delta=2\left[\mu(1)-\mu\left(1^{-}\right)\right]=U_{\text {eff }} \xi$.

In Fig. 4 we report the difference between the gap at finite $\lambda$ and that at $\lambda=0$ as a function of the adiabaticity ratio. Since the attraction between the electrons gets larger with increasing $\gamma$, the resulting Mott gap is more reduced. However in the strong $e l-p h$ coupling regime $(\lambda$ larger than 1) there is no dependence on the adiabaticity ratio since the particles localized by the strong correlation are small polarons. In fact in this regime the $M F$ solution at finite density is minimized for $f=1$ yielding the effective interaction $U_{\text {eff }}=U-2 g^{2} \omega_{0}$ for any finite value of adiabatic ratio. In the inset of Fig. 4 we show the variation of the effective interaction $U_{\text {eff }}$ as a function of the adiabaticity ratio making the comparison with the behavior of the Mott gap. In the regime where $\lambda<1$, the quantity $\xi$ affects the magnitude of the gap and its dependence on the adiabaticity ratio, while in the strong coupling regime $\xi \simeq 1$ implying that the gap in units of $W$ is just $U_{\text {eff }} / W=U / W-2 \lambda$.

In this work we have seen that in the metallic phase close to the Mott transition the spectral weight $Z$ is enhanced and the gap in the insulating Mott phase is reduced as $\lambda$ is raised. These behaviors can be related to some recent experimental and theoretical studies in $\mathrm{V}_{2} \mathrm{O}_{3}$ and $\mathrm{Cr}$-doped $\mathrm{V}_{2} \mathrm{O}_{3}{ }^{11.20 .21}$ where the Mott gap is unexpectedly small and in the metallic phase the quasiparticle peak in the photoemission spectrum has a significantly large weight in comparison with that theoretically predicted. We suggest that the inclusion of the $e l-p h$ interaction could be able to partially fill the discrepancies between the experimental observations and the theoretical studies. Clearly the single orbital model is not sufficient to fully explain the electronic and magnetic structure of such systems ${ }^{22}$ so a proper multi-orbital theory has to be considered in order to obtain a better agreement of the theory with experiments $\stackrel{21}{\underline{21}}$ The results due to the reduction of the effective repulsion caused by the $e l-p h$ coupling are valid when the lattice distortions are coupled to charge fluctuations like in the model of Eq.(11). However for general models the issue is delicate since there are interactions such as the Jahn-Teller coupling for which the phonon-mediated attraction could be even diminished by strong correlations ${ }^{23}$

Finally we can argue the modifications of the spectral properties at densities near the $M I$ phase and at halffilling just under the edge of localization. Clearly there is a finite spectral weight at the Fermi energy where quasiparticle states begin to appear. Therefore, in addition to the incoherent contribution at high energy, the spectral density presents also the coherent term. Close to the metal-insulator transition the coherent term could not be strongly affected by the $e l-p h$ coupling. In fact for the combined effect of the strong correlation and $e l-p h$ coupling the quasi-particle band can be narrower than the phonon energy $\omega_{0}$ implying the impossibility of the single phonon scattering between the quasi-particles. Therefore near the Mott transition the $e l-p h$ coupling affects mainly the incoherent term of the spectral density. This result is in agreement with recent experiments on $\mathrm{Bi}_{2} \mathrm{Sr}_{2} \mathrm{CaCu}_{2} \mathrm{O}_{8+\delta}$ made using angle-resolved photoemission spectroscopy ${ }^{\frac{9}{}}$ In fact it has been found that the oxygen isotope substitution mainly influences the broad high energy humps.

In conclusion, we have discussed the role of the $e l-p h$ interaction in modifying the physical properties of the electrons in the metallic phase close to the Mott transition and in the $M I$ phase. The approach to study the Holstein-Hubbard model has been based on a variational slave boson technique that provides results in agreement with $D M F T$. An analytic expression of the spectral density is derived in the Mott phase showing that due to the reduced effective repulsion the Mott gap decreases as the $e l-p h$ coupling constant increases. In this paper we have mainly discussed the phases without long-range order. The study of broken symmetry phases is possible within the slave-boson formalism ${ }^{4}$ and it is left for future work. 


\section{Figure captions}

F1 The phase diagram $U / W$ versus $\lambda$ at half-filling for $\gamma=0.2$ in the three-dimensional case. The transition lines separate the metallic state $M$ from the Mott insulator $M I$ and the bipolaronic insulator $B I$. The dotted line is the locus where $U-2 g^{2} \omega_{0}=0$. In the inset, the transition line between the metallic and Mott insulating phase is shown for different adiabaticity ratios $\gamma$.

F2 The difference between the spectral weight $Z$ at the Fermi energy (a) and the local magnetic moment $M$ at finite $\lambda(\mathrm{b})$ and their respective values at $\lambda=0$ for some values of the ratio $U / U_{c}$ in the three-dimensional simple cubic lattice.

F3 (a) The renormalized density of states $N$ (solid line) together with the dominant contribution $e^{-g^{2} f^{2}}$ $\tilde{A}(\omega)$ (dotted line) and the term due to the phonon replicas (dashed line) as function of the frequency $\omega$.

(b) The renormalized density of states $N$ for different values of the $e l-p h$ coupling constant $\lambda$ as a function of the frequency $\omega$.

F4 The difference between the gap at $\lambda=0$ and that at finite $\lambda$ in units of the bare half bandwidth $W$ as function of the adiabaticity ratio for different values of the $e l-p h$ coupling. In the inset the effective repulsion $U_{\text {eff }}$ and the Mott gap $\Delta$ as a function of the adiabaticity ratio $\gamma$.
1 N. F. Mott, Philos. Mag. 6287 (1961).

2 J. Hubbard, Proc. R. Soc. London Ser. A 276, 238 (1963).

3 M.C. Gutzwiller, Phys. Rev. 137, A1726 (1965); W. F. Brinkman and T. M. Rice, Phys. Rev. B 2, 4302 (1970).

4 G. Kotliar and A. E. Ruckenstein, Phys. Rev. Lett. 57, 1362 (1986).

5 J. W. Rasul and T. Li, J. Phys. C 21, 5119 (1988); M. Lavagna, Phys. Rev. B 41, 142 (1990).

6 R. Raimondi and C. Castellani, Phys. Rev. B 48, 11453 (1993).

7 A. Georges, G. Kotliar, W. Krauth, and M.J. Rozenberg, Rev. Mod. Phys. 68, 13 (1996).

8 R. J. McQueeney, J. L. Sarrao, P. G. Pagliuso, P. W. Stephens, and R. Osborn, Phys. Rev. Lett. 87, 77001 (2001).

9 G.-H. Gweon, T. Sasagawa, S.Y. Zhou, J. Graf, H. Takagi, D.-H. Lee, and A. Lanzara, Nature 430, 187 (2004).

10 A. J. Millis, Nature 392, 147 (1998); M. B. Salamon and M. Jaime, Rev. Mod. Phys. 73, 583 (2001).

11 G. Kotliar, Science 302, 67 (2003); P. Limelette, A. Georges, D. Jerome, P. Wzietek, P. Metcalf, and J. M. Honig, Science 302, 89 (2003).

12 T. Holstein, Ann. Phys. (Leipzig) 8, 325 (1959); ibid. 8, 343 (1959).

${ }^{13}$ W. Koller, D. Meyer, Y. Ono, and A. C. Hewson, Europhys. Lett. 66, 559 (2004); W. Koller, D. Meyer, and A. C. Hewson, Phys. Rev. B 70, 155103 (2004); G. S. Jeon, T.-H. Park, J. H. Han, H. C. Lee, and H.-Y. Choi, Phys. Rev. B 70, 125114 (2004).
14 H. Feshke, A. P. Kampf, M. Sekania, and G. Wellein, Eur. Phys. J. B 31, 11 (2003); H. Fehske, G. Wellein, G. Hager, A. Weisse, and A. R. Bishop, Phys. Rev. B 69, 165115 (2004).

15 A. Deppeler and A. J. Millis, Phys. Rev. B 65, 100301 (2002); A. Deppeler and A. J. Millis, cond-mat/0204617 M. Capone, G. Sangiovanni, C. Castellani, C. Di Castro, and M. Grilli, Phys. Rev. Lett. 92, 106401 (2004).

16 I. J. Lang and Yu. A. Firsov, Soviet Physics JETP 16, 1301 (1963); Yu. A. Firsov, Polarons (Moskow, Nauka, 1975).

17 V. Cataudella, G. De Filippis, G. Iadonisi, A. Bianconi, and N.L. Saini, Int. J. Mod. Phys. B 14, 3398 (2000).

18 C.A. Perroni, G. De Filippis, V. Cataudella, and G. Iadonisi, Phys. Rev. B 64, 144302 (2001) and references therein.

19 The spectral density is not shown for values of $\lambda$ close to 1 since the system shows phase separation at densities different from half-filling.

20 S.-K. Mo, J. D. Denlinger, H.-D. Kim, J.-H. Park, J. W. Allen, A. Sekiyama, A. Yamasaki, K. Kadono, S. Suga, Y. Saitoh, T. Muro, P. Metcalf, G. Keller, K. Held, V. Eyert, V. I. Anisimov, and D. Vollhardt, Phys. Rev. Lett. 90, 186403 (2003).

${ }^{21}$ G. Keller, K. Held, V. Eyert, D. Vollhardt, and V. Anisimov, Phys. Rev. B 70, 205116 (2004).

22 R. Shiina, F. Mila, F.-C. Zhang, and T. M. Rice, Phys. Rev. B 63, 144422 (2001).

23 J.E. Han and O. Gunnarsson, Physica B 292, 196 (2000). 


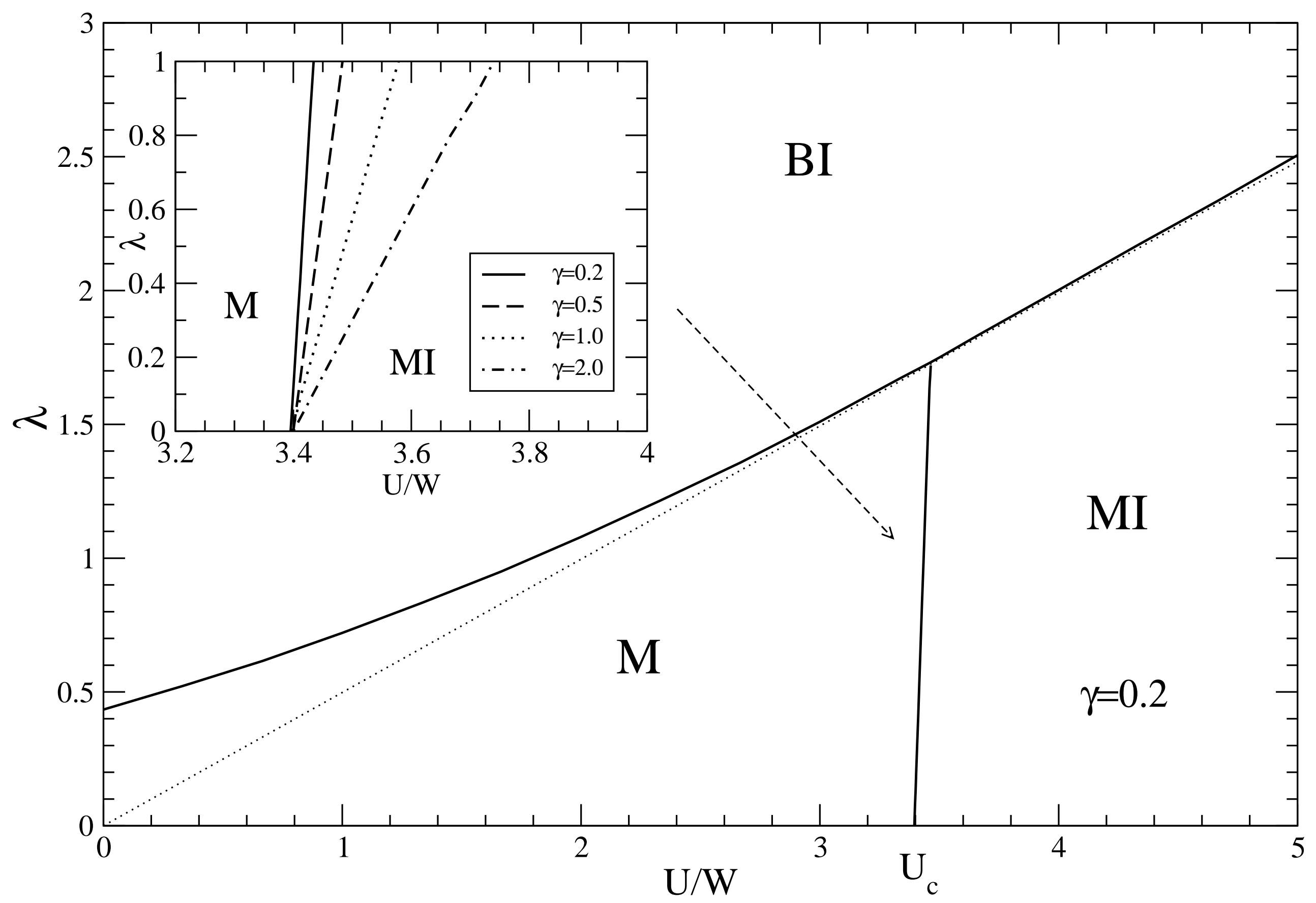



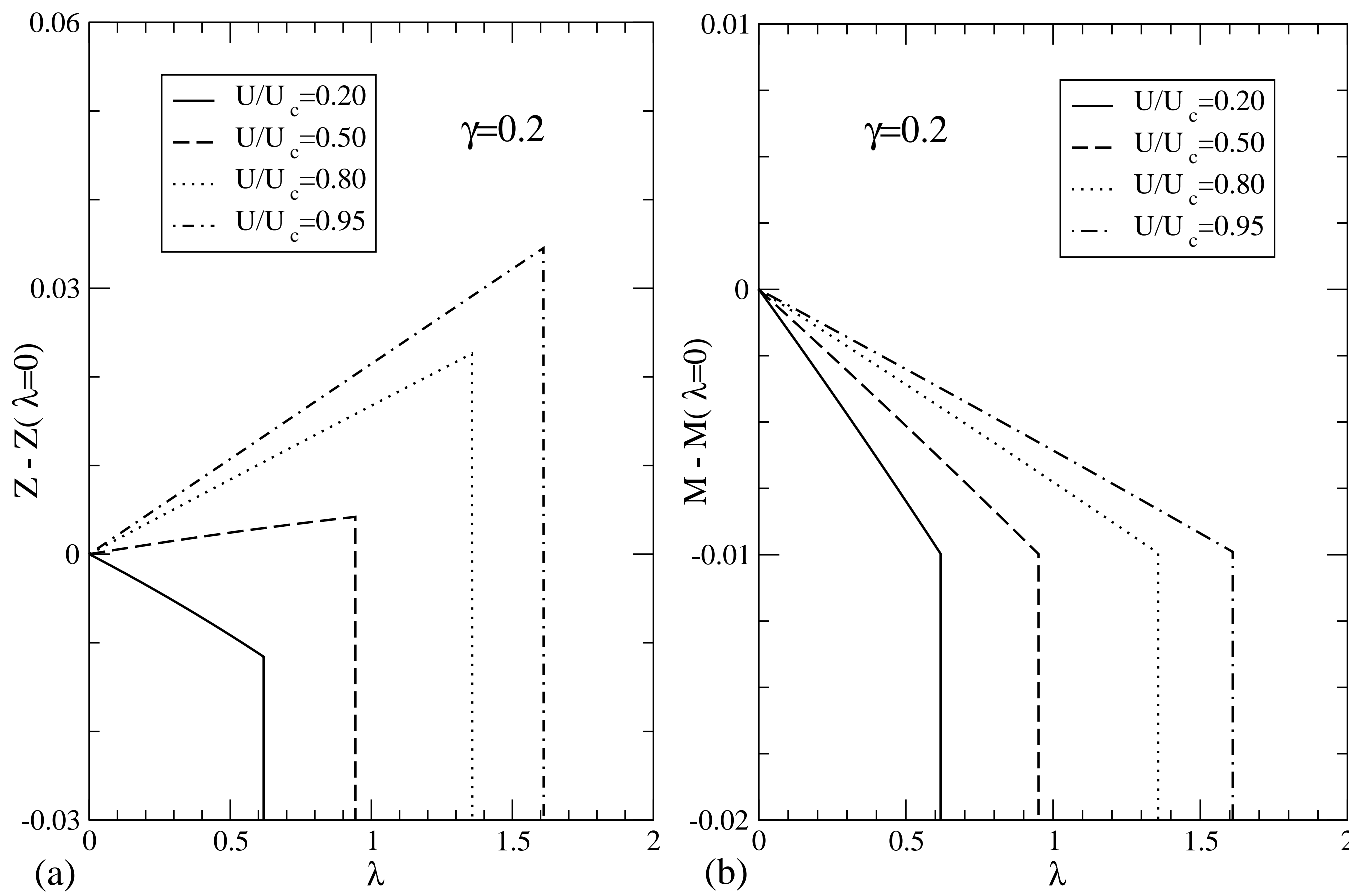

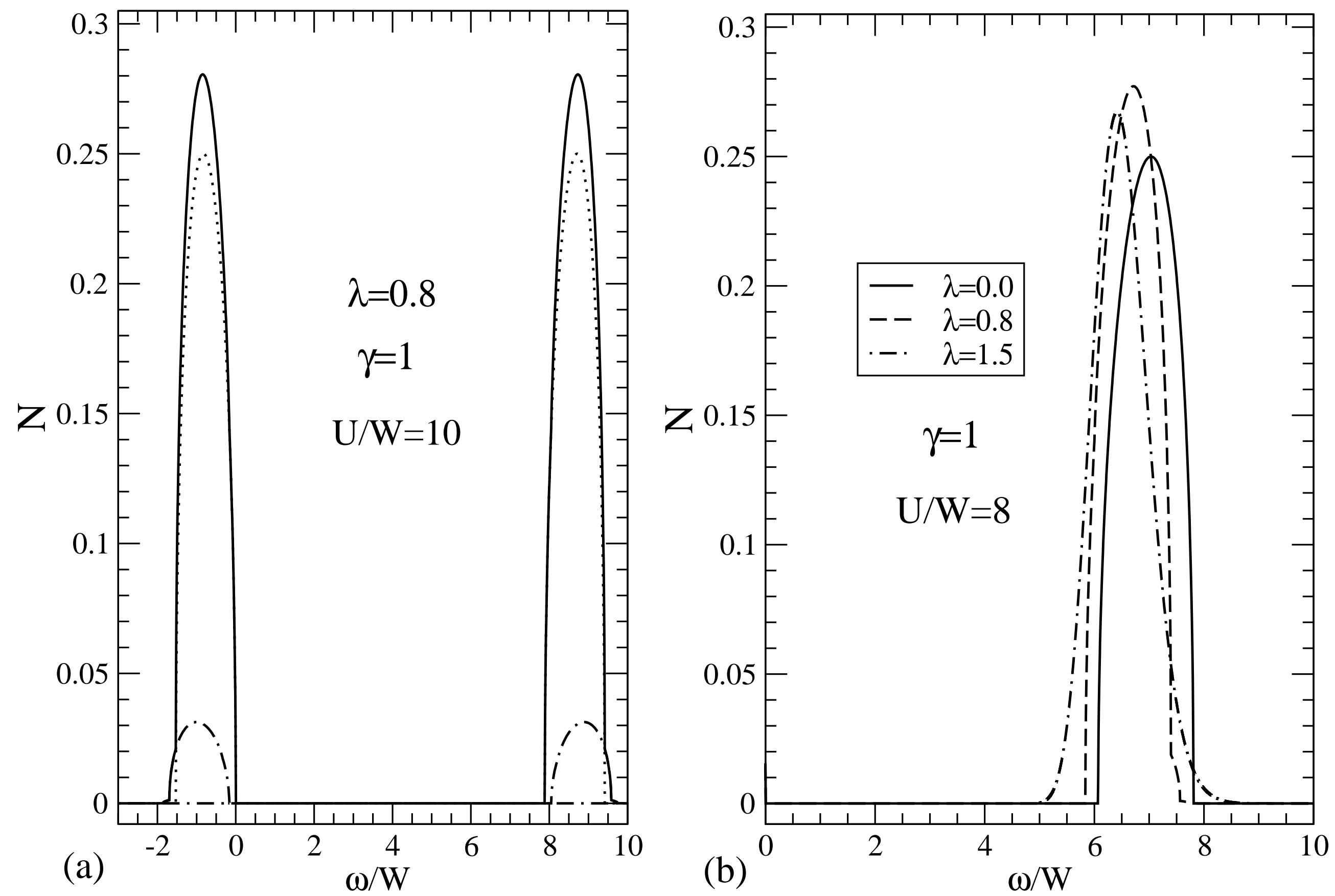


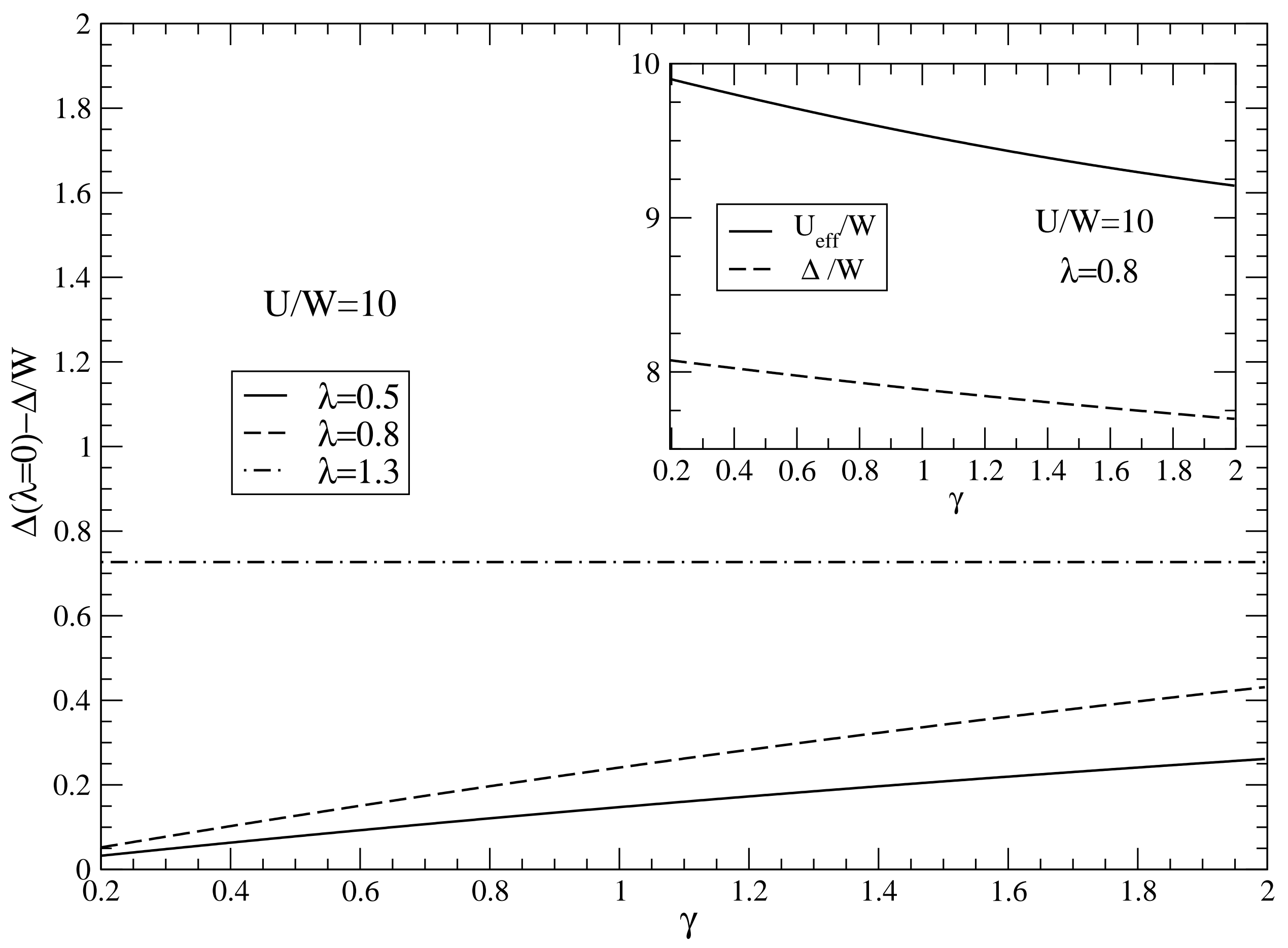

